\title{
Xylitol and Fluoride Provide an Alternative Preventive Therapy in Antibiotic Resistance Crisis
}

\section{Sunil Palchaudhuri", Anubha Palchaudhuri and Shreya Bhattacharyya}

Wayne State University School of Medicine, MI 48201, USA

${ }^{*}$ Corresponding author: Sunil Palchaudhuri, Wayne State University School of Medicine, MI 48201, USA, Tel: 011-91-33-24661637; E-mail: spalchau@med.wayne.edu

Received date: October 21, 2016; Accepted date: November 24, 2016; Published date: December 01, 2016

Copyright: (C) 2016 Palchaudhuri S, et al. This is an open-access article distributed under the terms of the Creative Commons Attribution License, which permits unrestricted use, distribution, and reproduction in any medium, provided the original author and source are credited.

Citation: Palchaudhuri S, Palchaudhuri A, Bhattacharyya S. Xylitol and Fluoride Provide an Alternative Preventive Therapy in Antibiotic Resistance Crisis. Med Clin Rev. 2016, 2:4.

\section{Abstract}

An alternative preventive treatment with low calorie five carbon sugar-alcohol (xylitol) in combination with fluoride has been developed. Xylitol phosphate affects the genetic continuity of all diplococcic Gram positive Streptococci are formed. Streptococci oralis (diplococcic) and Streptococci pneumoniae grow similarly in heterogeneity of their growth phases: Pre-competent, competent and postcompetent (cluster). If exposed to fluoride, such a cluster breaks apart with an instant increase of titre (there is a high probability to reach ID50 dose). We conclude that fluoride breaks clusters even in stationary phase; but the presence of xylitol phosphate initiates unfolding of bacterial cell walls (peptidoglycan layers) and terminates in their population of spheroplasts, protoplasts and PG fragments. Biological continuity ceases regardless of their antibiotic sensitivity patterns.

Keywords: Alcohol; Xylitol; Heterogeneity; Antibiotic; Mortality

\section{Introduction}

During the past 22 years, starting from 1993 there is an increasingly high mortality rate of children and the elderly from bacterial pneumonia and very recently WHO has declared that the penicillin and their newer derivatives are no longer effective against bacterial pneumonia. Even the newly developed polyvalent vaccine does not guarantee its success against such bacterium which is genetically capable of inducing serological variations [1]. Our data presented in this article demonstrates an alternative but preventive low-cost combination therapy in antibiotic resistance crisis.

If appropriately developed such a therapy should be effective to prevent all other severe diseases caused by the diplococcic Gram-positive bacterium Streptococci pneumoniae [2]. Diplococcic members of Mitis group bacteria if grown in rich broth containing xylitol (a low calorie five carbon sugar- alcohol), the xylitol phosphate is formed which directly affects bacterial signal transduction (biological reproduction). Incompetent phase population is defined as an irreversibly old population which is the target of bactericidal effect of its competent population, termed fratricidal effect [3]. On contrary our data opposes the above mentioned fratricidal effect. Bacterial population (diplococcic Streptococcus $s p$.) in heterogeneity when grown in rich broth (TSB or BHI), the nutrients get exhausted, and starvation begins and therefore the fraction of old population is autolysis. As an outcome the nutrients thus released are utilised to feedback the population in growth phase (pre-competent and competent).

In a recent article, we have shown that the Gram-positive bacteria S. oralis and S. pneumoniae (Mitis group) grow in heterogeneity of their growth phases: Pre-competent, competent and post-competent (growth curve) [4]. Interestingly, Dr Griffith's smooth and rough colonies of $S$. pneumoniae as reported in 1928 represent very much the competent and post competent phases of our growth curve. The pre-competent phase of our growth curve is the early logphase in which they grow only in size like human children. The round shape becomes oval with the time of growth and attains the competent phase [4]. In this phase, S. pneumoniae starts excreting pheromone and initiates bio-communication by one or two- component bio-signalling system $[5,6]$. They multiply by cell division and then suddenly they reach stationary phase when grown in liquid broth which is equivalent to rough colony when grown on blood agar solid medium. Rough colony (the colony with uneven shape) represents their heterogeneity of population (colony never means a single bacterium). It appears as clusters around the irreversibly old mother who has lost cell wall thickness (reduction of peptidoglycan layers). Such an old population is stained pink or pinkish like Gram negative Escherichia coli K-12 when visualized by the Gram staining technique. One should not think it is bacterial contamination as we have verified by using differential growth media like McKonkey lactose plate and CNA. The population of rough colony (stationary phase) if exposed to fluoride alone (200 ppm or less), their clusters break apart and therefore the bacterial titre (CFU) increases confirming that they prevail in chain. The concomitant presence of xylitol should not allow 
them to run away as the chain in which they prevail is stabilized irreversibly [7]. The phosphorylation of xylitol apparently forms the xylitol phosphate which initiates unfolding of cell wall Peptidoglycan Layers (PG). Such unfolding should end in spheroplasts/protoplasts with the cell wall PG fragments scattered around. These fragments have an inhibitory effect on the growth of Gram-positive Mitis group bacteria by interfering in the direct interaction of Stkp with the PBP2X [6].

We have already recognized antibiotic resistance crisis but recently some investigators have demonstrated such alterations of PBP genes (high molecular weights) occur only by point mutations [8]. The abuse and overuse of penicillin appears to be responsible for increasing the environmental stress, therefore, the increased infidelity of DNA replication $[9,10]$. For a dental pathogen Streptococci mutans, a combination therapy of xylitol with fluoride has been previously used but without much success [11]. Therefore, the knowledge of growth curve is essential and it justifies that use of fluoride by itself without the concomitant presence of xylitol is not safe at all. The S. oralis and S. pneumoniae have similar growth patterns and therefore their regulation by one/two component signalling system (bio-communication) is not expected to differ [6]. An alternative preventive low-cost therapy does neither require patients' body immunity nor does it depend on the antibiotic sensitivity pattern of the pathogen. If appropriately used, our proposed therapy should not only be able to prevent the disease pneumonia but few other severe diseases caused by the diplococcic Gram-positive Streptococci including dental caries.

\section{Materials and Methods}

Bacterial strains and experimental conditions Gram-positive diplococcic, catalase negative, non-hemolytic (gamma) and bile-esculin negative Streptococcus oralis (ATCC 6249) and a dental pathogen S. mutans (ATCC 25175) were used. These strains are saved in their separate stabs (mother stock). Bacteria were always genetically purified as a single colony isolate and then grown in the $\mathrm{BHI}$ broth containing dextrose 5 $\mathrm{g} / \mathrm{L}$ (DIFCO, Becton Dickinson) or in solid BHI-agar media (1.5\%). Whenever necessary, they were also grown at $37^{\circ} \mathrm{C}$ as well as at ambient temperature. Bacterial growth was monitored by counting the colony forming units on a $\mathrm{BHI}$ agar media after appropriate dilutions as well as by measuring optical density at $580 \mu \mathrm{m}$. It is a fastidious organism to grow in animal infection.

Gram-positive diplococcic Streptococcus overnight cultures of $S$. oralis were diluted 10,000 -fold in a rich broth TSB or $\mathrm{BH}$ by our previously described dilution procedure and grown at $37^{\circ} \mathrm{C}$ with or without shaking to saturation. Occasionally we have observed spontaneous lysis in our $24 \mathrm{~h}$ old cultures but not the cultures grown in the presence of $2 \%$ xylitol. Optical microscopy after Gram-staining technique with crystal violet and scanning electron microscopy has been used. Crystal violet solution was always prepared fresh and filtered through a sterile membrane filter disc $(0.2 \mathrm{um})$. Before starting experiments we had streaked overnight cultures on blood agar medium and the single colony transferred with sterile tooth picks onto CNA as well as MacConkey-lactose plates. Then the pure colony which grew only on CNA medium was used in our experiments. After shadowing with gold for 10-20 s, the sample was visualized by a scanning electron microscope, JEOL $\mathrm{JSM}-760^{\circ} \mathrm{F}$ at $15 \mathrm{kV}[12,13]$.

\section{Fluoride Experiments}

For one experimental set varying concentrations of fluoride: 0-300 ppm (NaF, Bio labs) were added to the overnight cultures of Streptococcus oralis without diluting the growth media. After addition of fluoride the bacteria were incubated at $22^{\circ} \mathrm{C}$ for $2-20 \mathrm{~min}$.

In another experimental set the overnight cultures of Streptococcus oralis were diluted 100 fold in fresh BHI medium containing xylitol with varying concentrations of xylitol. Xylitol stock solution ( $20 \%$ in $\mathrm{BHI})$, pre-warmed to $37^{\circ} \mathrm{C}$, was used to have varying concentrations of xylitol without diluting the growth media. The resulting cultures were incubated in a water bath at $37^{\circ} \mathrm{C}$ with shaking and samples were withdrawn at $1 \mathrm{~h}$ time intervals with varying concentrations of fluoride (0-300 ppm). The logarithmic growth-phase of the bacteria was monitored by counting the colonies on rich agar medium as well as by measuring optical density at $580 \mathrm{~nm}$. Gram-stain preparations of $S$. oralis were made after growing similarly in $\mathrm{BHI}$ medium. These experiments were repeated several times using the standard Gram-staining technique and optical microscopy (final magnification 1000-fold).

\section{Scanning Electron Microscopy (SEM)}

Bacterial culture (overnight or diluted 100 -fold in $\mathrm{BHI}$ broth containing $6.5 \%$ sucrose) was fixed on a mica sheet with $2.5 \%$ glutaraldehyde. Bacteria on the mica sheet were washed serially with $25 \%$ ethanol to absolute ethanol and the fixed bacteria were finally dried and shadowed with gold particles for $10 \mathrm{~s}$ to $20 \mathrm{~s}$. They were then seen by a scanning electron microscope (JEOL JSM $-760^{\circ} \mathrm{F}$ ) at $15 \mathrm{KV}$. The aliquot of the same bacterial sample has also been critically examined by optical microscopy following a standard Gram-staining procedure.

For each condition in this study at least 3 replicates were performed.

\section{Results}

\section{Heterogeneity of Streptococcus oralis}

The pre-competent phase population starts growing immediately after they are detached from their old parents but in heterogeneity of their sizes starting from $200 \mathrm{~nm}$ (mostly spherical and obviously this is an average size). They gradually attain adulthood (their competent phase) with elliptical shape (Figure 1a). We should make it clear that the irreversibly old population of $S$. oralis (incompetent ones still in-chains) remain surrounded by their pre-competent and competent progeny (clusters). Then clusters fall apart upon dilution in nutrient broth only pathogenesis differs but their 
genetic continuity remains the same. The population as shown in Figures $\mathbf{1 b}$ and $\mathbf{1 c}$ is not a homogenous population.

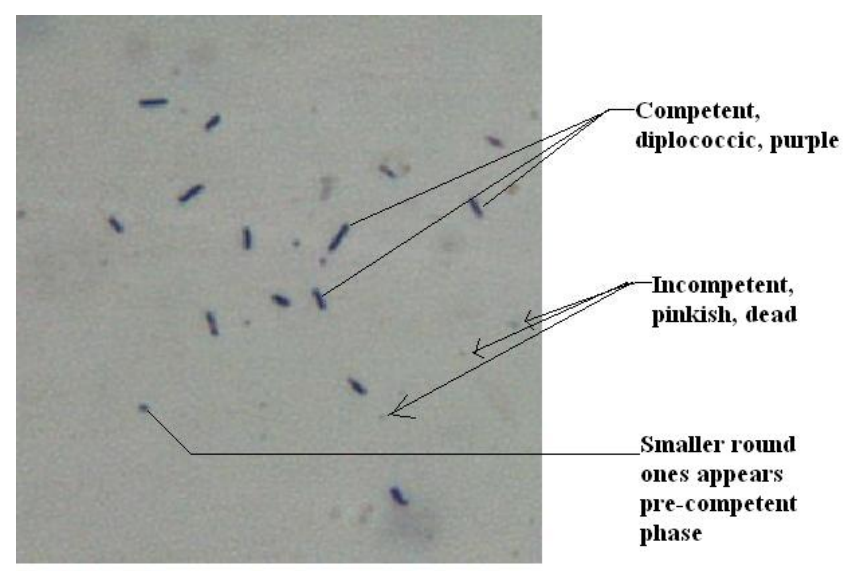

Figure 1a: Stationary phase viridans group streptococci diluted 10,000 -fold in rich broth, allowed to grow at $37^{\circ} \mathrm{C}$ and then visualized by optical microscopy after Gram staining (magnification 1000X). Bacterial population maintains heterogeneity of sizes including the precompetent, competent and the post-competent. Bacterial population in the post-competent phase are diminished because of the thinning of cell wall thickness (spheroplasts and protoplasts). If the growing membrane remains attached to the replicating chromosome but without the complete loss of cell-wall, they can still be visible. They can't be reverted back to their pre-competent phase. They show a mixture of purple and pink individuals with heterogeneity of sizes and colour if sample is prepared in the presence of sucrose $(6.5 \%)$ [12].

The incompetent old ones are pink (like Gram negative bacteria) but look much fragile apparently due to thinning of their cell wall thickness and much smaller in size (spheroplasts and protoplasts) but the stable purple ones range from 0.3 to 1.8 um appears minority in the total population. We have broken the chain and clusters by the shearing force induced by standard dilution techniques for the microscopy and therefore their clusters around old parents have been ruptured. There are also small purple ones, round in shape and we introduce them as their growing baby in the pre-competent phase [4].

They are dissociated from their parents under the environmental stress and apparently responsible for the breakage of their continuity. The purple ones appear much stronger than the pink, because of their cell wall PG difference. For further analysis we have visualized them under scanning electron microscope with low magnification (3000X) after shadowing for $20 \mathrm{~s}$ instead of $10 \mathrm{~s}$.

Previously, we have established how the presence of xylitol in their growth media contains the entire population in a long chain that includes their existence in different physiological states of growth. In order to visualize their prevailing in heterogeneity of growth phases, clusters in chains are fully ruptured by the exposure to fluoride (200 ppm) at different time intervals. When grown in absence of xylitol these chains fall apart proving bacterial growth phases in heterogeneity. Such growth in heterogeneity is termed clusters.

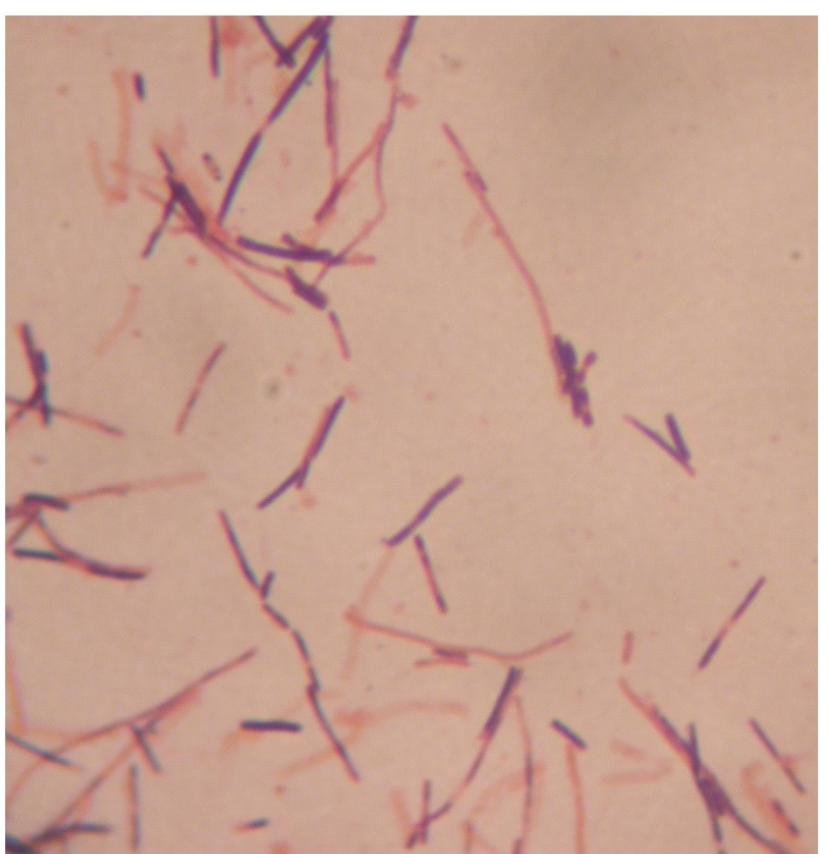

Figure 1b: Optical microscopy magnified $1000 \mathrm{X}$. The $S$. oralis population after $7 \mathrm{~h}$ growth or $24 \mathrm{~h}$ (stationary phase) is prepared for optical microscopy by a modified Gram staining technique with crystal violet. It confirms that stationary phase population represents the mixture of purple, pink and purple - pink combined in the same chain [12].

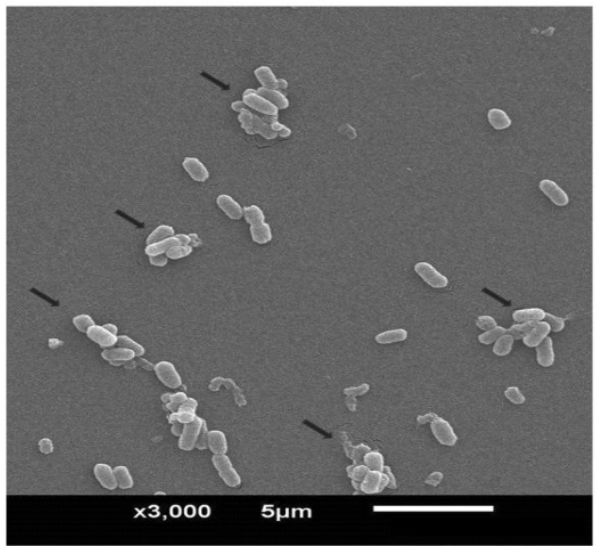

Figure 1c: Scanning electron microscopy of the same bacterial population, magnified 3000X Arrows indicate some clusters and some clusters in a chain. We have added sucrose to a final concentration of $6.5 \%$ after $7 \mathrm{~h}$ of growth to minimize breakage of their chains. We have selected one of these clusters/chains as marked by a rectangle and enlarged in our next figure (Figure 2a) for further analysis. 
The clusters are broken by the standard dilution techniques commonly used for measuring biological titre and the Gram staining technique is used for visualization based on the interaction with crystal-violet by optical microscopy.

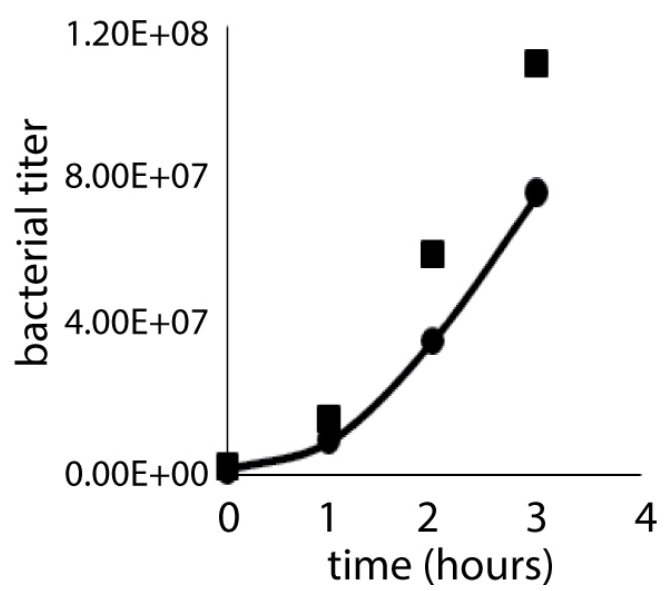

Figure 2a: Growth curve of S. oralis; Increase of S. oralistitre when the samples from log-phase culture are withdrawn at different time intervals and exposed to $100 \mathrm{ppm}$ fluoride (F). At $1 \mathrm{~h}$ time intervals, samples are withdrawn and exposed to fluoride (100 ppm) to assay the live CFU after overtaxing. Additional increase indicated with solid square means that these bacteria grow in clusters.

In order to minimize the breakage of chains, we have added sucrose to a final concentration of $6.5 \%$ before these bacteria are fixed in glutaraldehyde (2.5\%). Such existence in chains and in-clusters have been visualized by microscopy both optical and SEM after growth either in broths TSB, BHI or solid blood agar media.

$S$. oralis titre increases when exposed to fluoride at nonbactericidal doses ( $\mathrm{NaF}, 0-300 \mathrm{ppm}$ ).

Streptococcus mitis and S. oralis prevail in clusters around their old parents who are still in-chain when they have reached stationary or latent-phase. Therefore, the addition of fluoride at non-bactericidal doses breaks these clusters and results in a higher titre than the titre obtained without breaking the clusters. This is shown in Figure $\mathbf{2 a}$.

The samples are withdrawn at regular intervals and plated by diluting them in the $\mathrm{BHI}$ medium containing sucrose at $6.5 \%$. This high titre may satisfy the ID50 dose but the remedy is the presence of xylitol which stabilizes the entire population in their chains (we will address this issue in next paragraph). Figure $\mathbf{2 a}$ includes the $S$. oralis growth curve under standard conditions growth. Addition of fluoride (100 ppm) to a fixed volume of growing culture at different time intervals shows an increase of bacterial titre.

The bacterial titre due to the fluoride exposure increases with increasing time intervals, e.g., highest at $3 \mathrm{~h}$ relative to 0 min, $1 \mathrm{~h}$ or $2 \mathrm{~h}$ increments. Our interpretation is that these bacteria grow in chains of varying lengths. Therefore, when these bacteria are grown for longer time the chains keep growing longer. Our objective is to contain them stably in their chains by growing in the presence of xylitol.

Therefore, they should be resistant to fluoride or blunt the genetic ability to sense their immediate environment by pheromone which is necessary to attain competent-phase and bacterial reproduction (family planning). Without growth, the bacterial populations will not be able to develop antibiotic resistance crisis.

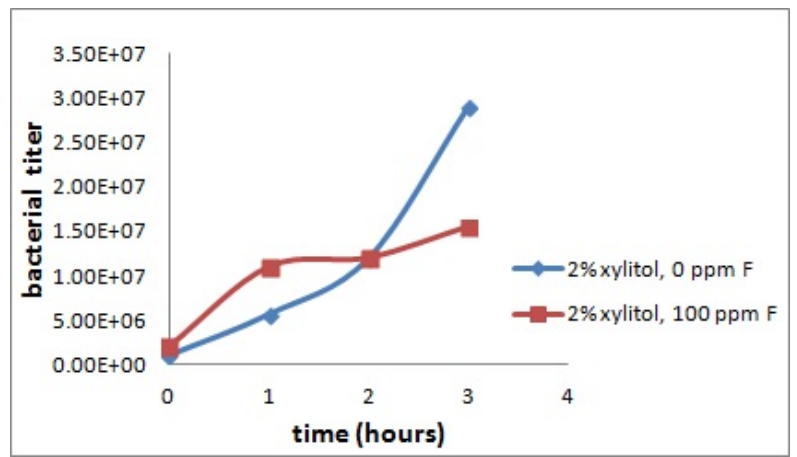

Figure 2b: If xylitol is simultaneously present with 100 ppm of fluoride. After two h of growth in the presence of xylitol, they are stabilized and exposure to fluoride does not show any additional increase. Because of the heterogeneity of their growth phases and the presence of fructose, the $100 \%$ of the population is not permeable to the entry of xylitol, the simultaneous exposure to fluoride and xylitol is preferred and alternatively repeated exposure to xylitol together with fresh nutrients may guarantee their success in such therapy.

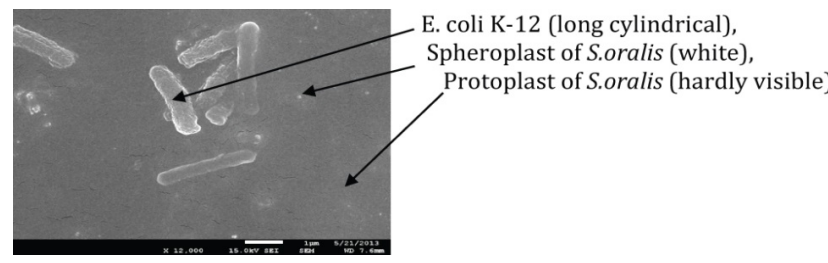

Figure 3: SEM of S. oralis grown in rich broth with xylitol (2.5\%). Gram negative E. coli K12 lacking xylitol loci (control) is mixed with $S$. oralis and grown together. The E. coli K12 ( 2.8 um) is distinctly visible together with spheroplasts and protoplasts of Gram positive S. oralis as formed by their growth in xylitol. Gradual unfolding of cell wall peptidoglycan layers (PG) appear as cloud around their protoplasts. Mag. $12000 \mathrm{X}$.

Morphological difference between S. oralis (xylitol+) and E. coli K-12(xylitol-)

Figure 3 shows if S. oralis (xylitol+) and E. coli K-12 (xylitol--) are grown simultaneously in the presence of xylitol, their morphological difference shows that Gram-negative $E$. coli K-12 don't change because it doesn't have locifor xylitol utilization. To the contrary, Gram-positive $S$. oralis (mitis group) gradually initiates the unfolding of cell wall 
peptidoglycan (PG). Obviously they will end in the formation of spheroplasts and protoplasts. To make it visible we had to have to overshadow for electron microscopy.

Spheroplasts and protoplasts of different sizes appear in the chain after the xylitol phosphate has unfolded their peptidoglycan layers

Figure 4 helps us to conclude that the unfolding begins at the outermost layer of the cell wall and moves inward until the protoplasts are formed in the course of growth in the presence of xylitol ( $2 \%$ or higher). Diminishing the cell wall thickness in the presence of xylitol (thinning) affects cleavage formed at the mid-cell position and hence the displacement of StkP and $\mathrm{PbP} 2 \mathrm{X}$. It has already been accepted that the bacterial signal transduction pathways involve protein kinases, proteinphosphatases and phosphoprotein-interacting domain (PASTA) to carry out regulation of biological activity [6].

Based on our data presented in a recent International Conference on Electron Microscopy we also think that the phosphorylation and de-phosphorylation of the serine/ threonine kinase are involved in allowing both Pneumococci and Mycobacteria to play hide and seek in their in vivo inhabitation [1]

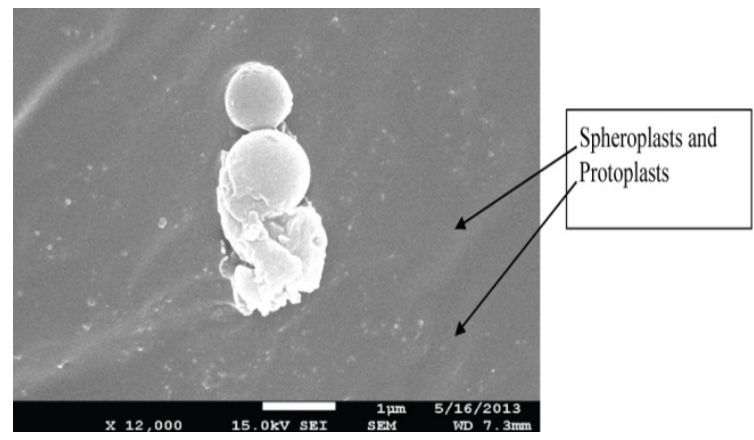

Figure 4: Arrows are showing spherotoplasts/protoplasts of S. oralis, still in clusters after xylitol phosphate has unfolded them of their Peptidoglycan Layers (PG) and converted into protoplasts (Magnification only 12000X). We find a diplococcic bacterium which has also been affected by their exposure to xylitol (seldom observed by SEM).

We now proudly announce that the xylitol phosphate once formed by these diplococcic bacteria initiates unfolding of their PG layers and affects bacterial reproduction. Therefore xylitol in combination with fluoride can be clinically applied to stop these Mitis group bacteria from killing our children. At the same time we have defined the rough colony and the heterogeneity of growth phases even in liquid broth and obviously the presence of xylitol can still be allowed but the use of fluoride alone (if not a bactericidal dose) is not at all a good idea for the safety of our children. We don't touch the bee hive during the day time or follow a procedure to contain them before we touch them!

The protoplasts formed are not stained by Gram staining technique but appears to be prevailing in their clusters as shown by arrowheads. The intensity differs because of their size difference which apparently differs in their interaction with gold particles which are scattered at random for creating a contrast by gold shadowing (technique used for visualization by SEM).

\section{Discussion}

Natural transformation is defined in this work as physiological changes initiating with the progeny of $S$. pneumoniae in their pre-competent phase. The pre-competent grows to become competent with their ability to excrete pheromone. We strongly believe such ability to biocommunicate initiates the ability to reproduce. Dr Griffith's rough colony and the post competent phase when visualized by SEM they confirm their growth in heterogeneity of their growth phases (pre-competent, competent and the incompetent, irreversibly old). Griffith's rough colony on blood agar medium formed after $24 \mathrm{~h}$ or longer is equivalent to the stationary phase when grown similarly in liquid broth. The Gram positive Mitis group Streptococci multiply but in clusters maintaining their genetic linkage with the old parents or just old mothers; therefore their comparison with Gram negative $E$. coli K-12 growth curve is inappropriate. We are very grateful to all these investigators and also to $E$. coli $\mathrm{K}-12$ for our knowledge in bacterial genetics but now for the last 22 years mortality rate of children from bacterial pneumonia is increasing at a very high rate even in the presence of penicillin, old and new. In fact, Berge et al. have shown the recruitment of single stranded nucleotides (donor DNA) with 3-prime $\mathrm{OH}$ end adhering at the cleavage site of the recipient but there seems to be no entry into the cytoplasm (13). Why not we draw a line that our miracle drug penicillin (discoverer and Nobel Prize winner Dr Fleming has never called it a miracle drug) is completely defeated by the diplococcic bacterium $S$. pneumoniae. We accept the truth that the exogenous DNA of S. pneumonie even in single stranded form does not play any role during bacterial physiological changes like $E$. coli $\mathrm{K}-12$. We want to conclude the formation of xylitol phosphate is a preventive, low- cost therapy which should stop the diseases of our children and the elderly from bacterial pneumonia, otitis media, meningitis and dental caries even in the presence of penicillin resistance crisis.

\section{Acknowledgement}

Professor SP has designed and done all the experiments, Dr AP has shared her knowledge of medical Microbiology. SB has worked as a part-time Academic Assistant during Professor SP's stay in India.

\section{References}

Salyers AA, Whitt DD (2002) Bacterial Pathogenesis: a molecular approach, Edition 2, ASM Press, Washington, D.C.

Palchaudhuri S, Palchaudhuri A, Chatterjee B (2015) Xylitol in prevention of dental caries and respiratory disease caused by Diplococcic streptococcus. Arch Med Microbiol 6: 4. 
3. Havarstein LS, Coomaraswamy G, Morrison DA (1995) An unmodified heptadecapeptide pheromone induces competence for genetic transformation in Streptococcus pneumoniae. Proc Natl Acad Sci USA 92: 11140-11144.

4. Palchaudhuri S, Palchaudhuri A, Chatterjee B (2016) Growth curve of Streptococcus oralis. EJBR 6: 36-41.

5. Ruggiero $A$, De Simone $P$, Smaldone $G$, Squeglia $F$, Berisio $R$ (2012) Bacterial cell division regulation by Ser/Thr kinases: a structural perspective. Curr Protein Pept Sci 13: 756-766.

6. Morlot C, Bayle L, Jacq M, Fleurie A, Tourcier G, et al. (2013) Interaction of Penicillin-Binding Protein $2 x$ and Ser/Thr protein kinase StkP, two key players in Streptococcus pneumoniae R6 morphogenesis. Mol Microbiol 90: 88-102

7. Palchaudhuri S, Rehse SJ, Hamasha K, Syed T, Kurtovic E, et al. (2011) Raman spectroscopy of xylitol uptake and metabolism in Gram-positive and Gram-negative bacteria. Appl Environ Microbiol 77: 131-137

8. Maurer P, Todorova K, Sauerbier J, Hakenbeck R (2012) Mutations in Streptococcus pneumoniae penicillin-binding protein 2x: importance of the C-terminal penicillin-binding protein and serine/threonine kinase-associated domains for beta-lactam binding. Microb Drug Resist 3: 314-321

9. Lacks SA (1989) Generalized DNA mismatch repair - its molecular basis in Streptococcus pneumonia and other organisms. In: Butler LO, Harwood C, and Moseley BED (eds.) Genetic Transformation and expression. Intercept Andover, England pp: 325-339.

10. Tippin B, Pham P, Goodman MF (2004) Error-prone replication for better or worse. Trends Microbiol 12: 288-295.

11. Maeharaa H, Iwami Y, Mayanagi H, Takahashi N (2005) Synergistic Inhibition by Combination of Fluoride and Xylitol on Glycolysis by Mutans Streptococci and Its Biochemical Mechanism. Caries Res 39: 521-528.

12. Czarnecki G, Dissanayake P, Palchaudhuri S (2013) Gram positive S. mitis become sensitive to colistin and nalidixic acid when grown in Xylitol. J Mol Genet Med 7: 4172 -4177

13. Berge MJ, Kamgoue A, Martin B, Polard P, Campo N, et al. (2013) Midcell recruitment of the DNA uptake and virulence nuclease, End A, for Pneumococcal transformation. PLoS Pathog. 